\title{
Lipids and membrane lateral organization
}

\author{
Sandro Sonnino and Alessandro Prinetti*
}

Department of Medical Chemistry, Biochemistry and Biotechnology, University of Milano, Milano, Italy

Edited by:

Manuel Jose Prieto, Instituto Superior Técnico, Portugal

\section{Reviewed by:}

Nicoletta Kahya, University Medical

Center Groningen, Netherlands

Luis M. S. Loura, University of

Coimbra, Portugal

\section{${ }^{*}$ Correspondence:}

Alessandro Prinetti, Dipartimento di Chimica, Biochimica e Biotecnologie per la Medicina, Via Fratelli Cervi 93,

20090 Segrate, Milano, Italy.

e-mail, alessandro.prinetti@unimi.it
Shortly after the elucidation of the very basic structure and properties of cellular membranes, it became evident that cellular membranes are highly organized structures with multiple and multi-dimensional levels of order. Very early observations suggested that the lipid components of biological membranes might be active players in the creation of these levels of order. In the late 1980s, several different and diverse experimental pieces of evidence coalesced together giving rise to the lipid raft hypothesis. Lipid rafts became enormously (and, in the opinion of these authors, sometimes acritically) popular, surprisingly not just within the lipidologist community (who is supposed to be naturally sensitive to the fascination of lipid rafts). Today, a PubMed search using the key word "lipid rafts" returned a list of 3767 papers, including 690 reviews (as a term of comparison, searching over the same time span for a very hot lipid-related key word, "ceramide" returned 6187 hits with 799 reviews), and a tremendous number of different cellular functions have been described as "lipid raft-dependent." However, a clear consensus definition of lipid raft has been proposed only in recent times, and the basic properties, the ruling forces, and even the existence of lipid rafts in living cells has been recently matter of intense debate. The scenario that is gradually emerging from the controversies elicited by the lipid raft hypothesis emphasizes multiple roles for membrane lipids in determining membrane order, that encompass their tendency to phase separation but are clearly not limited to this. In this review, we would like to re-focus the attention of the readers on the importance of lipids in organizing the fine structure of cellular membranes.

Keywords: lipid rafts, phase separation, sphingolipids, cholesterol

\section{INTRODUCTION}

It would be difficult to imagine life without biological membranes. Biological membranes provide physical boundaries between different worlds, separating the cellular and extracellular environments and the diverse cellular compartments. However, they are highly dynamic and interactive boundaries, as they act as a scaffold for molecules and molecular complexes that physically and functionally link these different environments (Singer and Nicolson, 1972).

The fundaments for the structure and functions of biological membranes are determined by the intrinsic properties of membrane lipids. Ever since 1925, it became clear that membranes are a bimolecular sheet (Gorter and Grendel, 1925), and at the end of the 1960s the nature of this bimolecular sheet as a bilayer of amphipathic lipids was unveiled (Steim et al., 1969; Engelman, 1970; Tourtellotte et al., 1970). Thus, the creation of the lipid bilayer, a consequence of the aggregational properties of complex amphipathic membrane lipids, represents the first level of lipid-driven organization in biological membranes. To serve its primary function as a physical boundary, the lipid bilayer as a whole is a very stable structure. However, the fatty acyl chains of the phospholipids constituting the bulk of biological lipid bilayers at $37^{\circ} \mathrm{C}$ are in a fluid phase. Thus, biological lipid bilayers at physiological temperature are bidimensional fluids. The fluid mosaic model proposed by Singer and Nicolson (1972) describes the fluid phospholipid bilayer as the solvent for membrane proteins, implying that, as for a three-dimensional viscous solution, the protein molecules dissolved in the two-dimensional fluid would possess a certain degree of lateral motility, freely diffuse in the phospholipid bilayer, and distribute along the membrane surface in a random ("aperiodic") arrangement. The fluid mosaic model, implying a random distribution of plasma membrane components over microscopic distances, predicts the absence of long-range order in plasma membranes. However it assumes that some of the lipid components might be not in the bulk, fluid bilayer phase, but they might rather be more strongly (specifically?) interacting with the membrane proteins, thus allowing lateral heterogeneity on a short distance $(<100 \mathrm{~nm})$. In addition, the model incorporates the notion that the random distribution of membrane components might be perturbed by external, physiological stimuli, and that the subsequent "aggregation" of membrane components might be of crucial importance in some biological processes.

Shortly after the fluid mosaic model had been formulated (19741978), experiments studying thermal effects on the behavior of membrane lipids suggested that phase behavior of lipid mixtures could be responsible for a certain degree of lateral organization in biological membranes, introducing the notion that the collective aggregational properties of membrane lipids might be the driving force for the creation of a second level of order in biological membranes (Lee et al., 1974; Wunderlich et al., 1975, 1978). In 1982, the concept that the existence of multiple phases in the membrane lipid environment can drive the "organization of the lipid components of membranes into domains" (Karnovsky et al., 1982) was clearly formulated. This concept became the basis of the lipid raft hypothesis (Simons and van Meer, 1988). However, while there is today no doubt that "ordered structures that differ in lipid and/or protein composition from the surrounding membrane" (to this writer, 
this is the minimal acceptable definition for membrane domains; Lindner and Naim, 2009) exist in biological membranes, the real contribution of phase separation to the stabilization of membrane domains in living cells is just beginning to be elucidated.

This is probably not surprising, keeping in mind the huge diversity of membrane lipids. A typical biological membrane contains hundreds of different lipid species, as progressively elucidated by the emerging contribution of a sophisticated "lipidomic" approach. We have detailed information on the phase behavior for only relatively simple mixtures of the most common membrane lipids (Sonnino et al., 2006; Goni et al., 2008; Quinn, 2010). Translating this information to the phase behavior of natural membranes has proven to be extremely difficult, and a reductionist approach might be conceptually inadequate to investigate a phenomenon that is based on the maintenance of collective properties (this concept has been nicely addressed in a recent review (Mouritsen, 2010). In addition, our capability to understand the importance of lipid phase separation in organizing membranes has been further limited by other factors: (1) the complexity of membrane chemistry is not limited to lipid components. Biological membranes do contain an incredible number of different proteins (maybe it is worth to recall that, when the fluid mosaic model was proposed, the notion that one single type or class of "structural" membrane protein exists had been just recently refuted). Membrane proteins can interact with lipids, or can partition between different lipid phases. Thus, it is clear that other lipid-driven or lipid-influenced interactions, in addition to phase separation, can be extremely relevant to determine membrane organization; (2) biological membranes are systems not at equilibrium (Mayor and Rao, 2004), while most pieces of information regarding lipid phase separation have been obtained from the study of equilibrium artificial systems. Many studies on lipid rafts in biological systems rely on the putative resistance of lipid raft components to the solubilization by non-ionic detergents. Detergent-resistant membrane preparations might reflect the properties of lipid rafts in living cells (Sonnino and Prinetti, 2008), however they are undoubtedly systems driven to equilibrium by the specific experimental conditions used for detergent extraction systems. To understand the dynamics (in time and space) of phase separation-driven domains in living cells we still miss adequate experimental tools, even if the first pieces of information in this direction have been recently provided.

\section{FLUID-FLUID PHASE SEPARATION AS DRIVING FORCE FOR MEMBRANE HETEROGENEITY}

The pillar of the original lipid raft model is that lipids can organize domains in cellular membranes. This ability is due to the limited solubility of lipid in lipid mixtures. The non-complete miscibility of lipids in complex mixtures can be described using phase diagrams (Goni et al., 2008). This property leads to phase separation in model systems as well as likely in biological membranes (Sonnino et al., 2006, 2007; Lindner and Naim, 2009; Westerlund and Slotte, 2009; Elson et al., 2010; Mouritsen, 2010; Quinn, 2010). It is clear that in cell membranes, in addition to liquid-liquid immiscibility, heterogeneity can be driven by a multitude of specific lateral interactions. Then, why phase separation should be biologically relevant? Phase separation can be observed in all membrane models that recapitulate the other basic properties of a cellular membrane; moreover, the main difference between bona fide lipid rafts and the surrounding membrane is the lipid composition. On this basis, lipid rafts should be defined as areas of phase separation in biological membrane (Lindner and Naim, 2009), the properties of lipid rafts in living cells should be dictated by the forces governing phase separation, and a biological event should be lipid raft-dependent if dependent on those forces. Since phase separation of lipids is a consequence of the collective properties of a membrane lipid environment, the dependence of a biological event on lipid rafts should not be defined on the basis of natural or experimental changes in the cellular levels of a single lipid (in other word, a biological event or cellular properties can be dependent on a specific lipid, e.g., cholesterol-dependent, without being lipid raft-dependent).

Lipid bilayers usually exist in a liquid-disordered (1d) phase characterized by high fluidity, in which the lipid acyl chains are disordered and highly mobile. Lowering the temperature below the melting point freezes the lipid acyl chains in an ordered gel phase (solid-ordered) with very limited freedom of movement. In mixtures comprising a bilayer-forming lipid, such as dipalmitoylphosphatidylcholine, and cholesterol (or ergosterol in yeast), a third physical phase, the liquid-ordered (lo) phase (Ipsen et al., 1987), can be observed. In the lo phase, the acyl chains of lipids are extended and ordered, as in the gel phase, but have higher lateral mobility in the bilayer.

Membrane complex lipids are highly heterogeneous in their hydrophobic portions. The prevalence of glycerophospholipids containing unsaturated acyl chains ensures the fluidity of biological bilayers. However, glycerolipids with saturated chains are not neglectable membrane components (Prinetti et al., 2001), and in some other classes of complex membrane lipids (e.g., sphingomyelin and gangliosides, at least in the nervous system), palmitic and stearic acid represent the main fatty acids. Lipids with a high content of saturated acyl chains (that possess a high melting point and can be tightly packed with a high degree of order in the hydrophobic core of a bilayer) are characterized usually by a higher transition temperature, within or above the physiological range (Prinetti et al., 2001; Sonnino et al., 2006). The difference of transition temperature due to the different acyl chain composition probably represents one of the main forces leading to phase separation in lipid mixtures and aggregates, including bilayers. Indeed phase separation can be observed in binary mixtures of diacyl lecithins differing in chain length and/or saturation (Goins et al., 1986; Masserini and Freire, 1986; Masserini et al., 1988, 1989; Rock et al., 1991; Terzaghi et al., 1993; Palestini et al., 1994, 1995) and, in turn, complex lipids containing palmitic acid are highly enriched in detergent-resistant membrane fractions from cells (e.g., neurons) (Prinetti et al., 2001; Pitto et al., 2002).

Phase separation of sphingomyelin in dimyristoylphosphatidylcholine bilayers depends on the degree of sphingomyelin chain mismatch (Kahya et al., 2005) and distribution of ganglioside GM1 in the fluid phase of a phospholipid bilayer (Palestini et al., 1995) is inversely correlated with the acyl chain length and directly correlated with the degree of unsaturation. Brain gangliosides, usually highly enriched in stearic acid, are typical lo phase lipids. On the other hand, very long $(\geq \mathrm{C} 24)$ fatty acids are abundant in sphingolipids outside the nervous system. Lipid bilayers of the skin stratum corneum, characterized by an extremely high content of unusually long 
chain ceramides, are very rigid (Bouwstra and Ponec, 2006), and, based on neutron diffraction experiments on artificial membranes, it has been proposed that the organization of stratum corneum lipid bilayers could be stabilized by a partial interdigitation between the two leaflets (Ruettinger et al., 2008). Interdigitated hydrocarbon chains seem to play a role in the stabilization of lipid domains in human neutrophils, enriched in lactosylceramide with a high content of C24 fatty acid chains (Iwabuchi et al., 2008; Yoshizaki et al., 2008). Interdigitation of long chain fatty acid residues of complex membrane lipids might thus represent a further feature that favors the separation of phases with a higher level of order. This hypothesis still needs to be proven, however, it has been suggested that, even in the absence of interdigitation, long chain fatty acid-containing sphingolipids can form quasi-crystalline structures in glycerophospholipid bilayers (Quinn, 2010). Cholesterol, that alone has a melting point of $148.5^{\circ} \mathrm{C}$, preferentially associates with ordered acyl chains of complex lipids, due to the tight packing of the planar smooth $\alpha$-face of the sterol ring against the extended acyl chains of 10 phase lipids, both glycerol- and sphingolipids (Mouritsen, 2010; Quinn, 2010). Cholesterol (in a wide molar range, including also physiologically reasonable concentrations) forms a liquid-ordered phase in dimyristoylphosphatidylcholine or distearoylphosphatidylcholine bilayers (Almeida et al., 1992) alone, in phospholipid bilayers in the presence of sphingomyelin, that mixes more ideally with cholesterol that a phosphatidylcholine with the same acyl chain (Snyder and Freire, 1980; Sankaram and Thompson, 1990) and in sphingomyelin vesicles (Ferraretto et al., 1997). In the lo phase, the sterol molecules are tightly intercalated between the ordered acyl chains of the bilayer-forming lipid (Brown and London, 2000; Sonnino et al., 2006), and cholesterol is usually regarded as a key lipid component of lipid rafts. It should be noted that despite this, understanding the real role of cholesterol in stabilizing membrane domains is made difficult by the lack of precise information about the transbilayer distribution of this molecule. Reports in the literature indicate that a large fraction of plasma membrane cholesterol is associated with the inner leaflet $(75 \%$ in human erythrocytes, Schroeder et al., 1991; 85\% in mice synaptic membrane, Igbavboa et al., 1997). Ergosterol, the main sterol in fungi, is also able to stabilize liquid-ordered phases (Klose et al., 2010). Coexistence of $1 o$ and $l d$ phases has been shown in ternary mixtures of cholesterol with a high- and a low-transition temperature lipid (for example, in ternary mixtures of dipalmitoyl PC, dioleyl PC, and cholesterol (Veatch and Keller, 2005), sometimes using a sphingolipid as high melting lipid (Mattjus and Slotte, 1996). A strong preferential interaction between cholesterol and sphingomyelin, leading to the formation of liquid-condensed cholesterol- and sphingomyelin-rich domains, has been suggested by some studies in mixed monolayers (Li et al., 2003; Mattjus and Slotte, 1996; OhvoRekila et al., 2002). However, this notion has been subsequently confuted by a paper (Holopainen et al., 2004) clearly showing that in phospholipid bilayers there is no evidence of such a specific interaction between cholesterol and sphingomyelin.

In the case of sphingolipids, phase separation and association with lo phase in glycerophospholipid bilayers are favored by two additional features, that are unique to this class of lipids. (1) Sphingolipids, as ceramide-based amphipathic lipids, can create a complex network of hydrogen bonds thanks to the presence in the ceramide moiety of the amide nitrogen, the carbonyl oxygen and the hydroxyl group positioned in proximity of the water/ lipid interface of the bilayer (Pascher, 1976). The contribution of a hydrogen bond network to lipid-lipid interactions stabilizing a more rigid segregated phase in the bilayer is energetically remarkable (3-10 kcal per hydrogen bond vs $2-3 \mathrm{kcal}$ per interaction in the case of van der Waals forces between hydrocarbon chains). The relevance of this factor has been confirmed by recent studies showing that (a) mixtures of natural sphingomyelin and phosphatidylcholine molecular species with comparable fatty acyl chains are largely immiscible at temperatures above the transition temperature of sphingomyelin (Quinn and Wolf, 2009); (b) increased order can be observed in C18sphingomyelin molecules in a dioleylphosphatidylcholine bilayer due to the formation of sphingomyelin nanoclusters stabilized by hydrogen bonds (Mombelli et al., 2003; Pandit et al., 2004). (2) Glycosphingolipids, present in all mammalian cell membranes as minor components, however abundant in some tissues (e.g., brain) and cell types (e.g., neurons) and asymmetrically enriched in the outer leaflet of the plasma membrane, are defined on the basis of their sugar hydrophilic headgroup. Even the simplest, monosaccharide-based glycosphingolipid headgroup is much bulkier that phospholipid headgroups, and, as a general trend, the volume occupied by the sugar headgroup increases with the complexity of the oligosaccharide chain (Figure 1). Theoretical calculations of minimum energy conformation show that the oligosaccharide hydrophilic headgroup of ganglioside GM1, one of best studies gangliosides, occupies a volume much larger than that occupied by phosphocholine, the bulkiest headgroup present in phospholipids (Acquotti et al., 1990). Predictions based on the geometrical properties of glycosphingolipid molecules indicated that separation of a glycosphingolipid-rich phase in a phospholipid bilayer, concomitantly accompanied by the acquisition of a positive membrane curvature, would imply a minimization of the interfacial free energy required to accommodate the amphipathic glycosphingolipid molecule in the bilayer. In other words, the geometrical properties dictated by the bulky hydrophilic headgroup of glycosphingolipid strongly favor phase separation and spontaneous membrane curvature (Acquotti et al., 1990, 1991, 1994; Scarsdale et al., 1990; Sonnino et al., 1990a,b, 1994; Levery, 1991; Siebert et al., 1992; Poppe et al., 1994; Brocca et al., 1995, 1996,

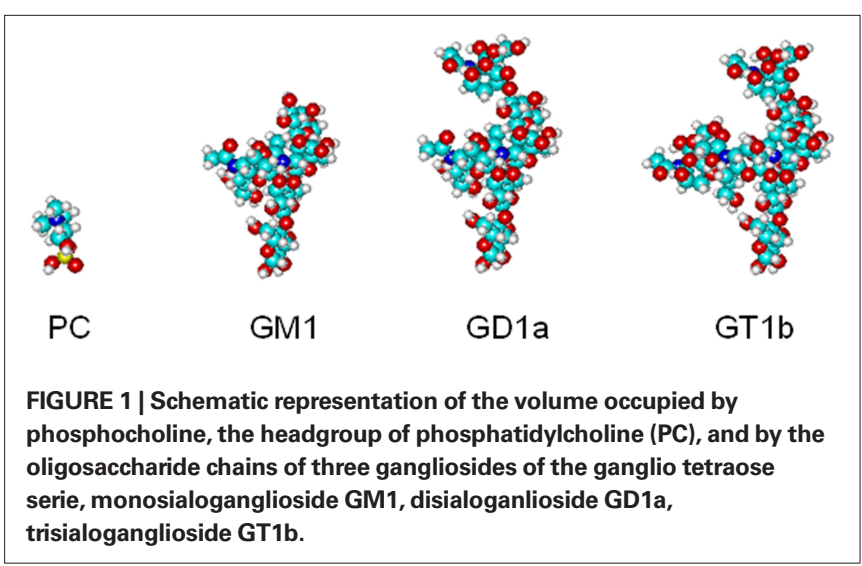


1998). These predictions are confirmed by the observation that the extent of ganglioside phase separation in glycerophospholipid bilayers depends on the surface area occupied by the glycosphingolipid oligosaccharide chain, that is usually directly correlated with the number of sugar residues present in the oligosaccharide (Masserini and Freire, 1986; Masserini et al., 1988, 1989). In addition, GM1-enriched domains can be formed in sphingomyelin bilayers (Ferraretto et al., 1997) and phase separation was observed in mixed micelles of two different gangliosides (GM2 and GT1b (Cantu et al., 1990), GD1b and GD1b-lactone (Cantù et al., 1991), or GM1 and GD1a (Del Favero et al., 2010) with identical composition of the hydrophobic moiety (Figure 2).

It has been recently proposed that membrane curvature can greatly contribute to the reduction of line tension (the energy required to maintain a border between a membrane domain and the surrounding membrane environment) (Baumgart et al., 2003), thus representing a general principle explaining the segregation of lipids and proteins in cellular membranes (Tian and Baumgart, 2009).

It has been suggested that clustering of glycosphingolipids could be further stabilized by the formation of lateral carbohydrate-carbohydrate interactions. Nevertheless, while head-to-head carbohydrate-carbohydrate interactions have been convincingly described for glycolipids (Hakomori, 2004), direct side-by-side oligosaccharide-oligosaccharide interactions remain at present merely hypothetical, and NMR studies on ganglioside micelles (Brocca et al., 1998) seem to exclude significant inter-molecular side-by-side interactions. On the other hand, NMR revealed strong interactions between different portions of GM1 oligosaccharide and solvent water molecules (Brocca et al., 1998) suggesting that a network of water-mediated hydrogen bridges could contribute to the stabilization of a glycolipid cluster (it is here worth to recall that water bridges between saccharides play a relevant role in stabilizing the tridimensional structure of hyaluronan (Heatley and Scott, 1988).

\section{LIQUID-ORDERED PHASE IN REAL BIOLOGICAL MEMBRANES?}

Altogether, studies in relatively simple membrane model systems strongly suggest that fluid-fluid immiscibility, with the separation of a liquid-ordered phase, provides in principle an explanation for

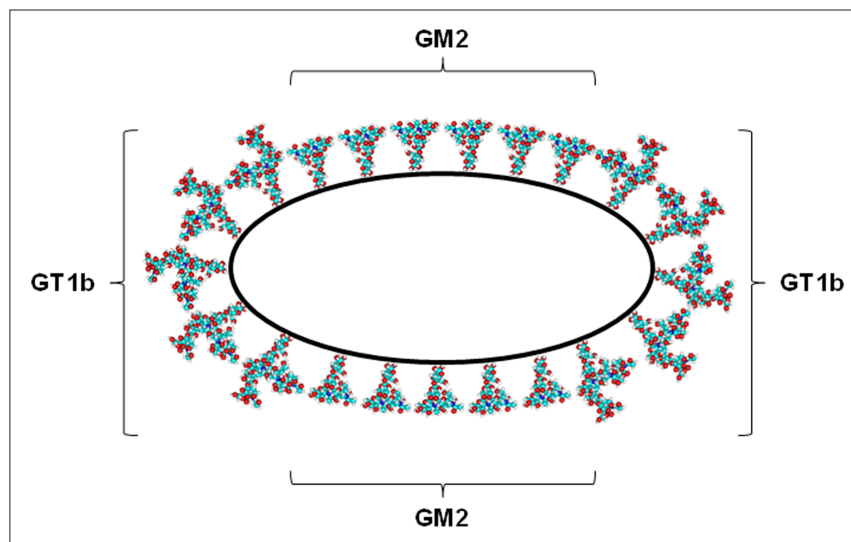

FIGURE 2 | Schematic representation of phase separation driven by the differences in the oligosaccharide chains in a GM2/GT1b micelle. the existence of membrane heterogeneity as a consequence of the intrinsic properties of the lipid components of biological membranes. The main criticism against this view is represented by the fact that model systems represent a strong simplification respect to the complexity of the lipid environment in a real membrane. Thus, a big effort has been made to extend these studies to systems closer to the complexity of biological membranes, and fluid phase separation has been observed in reconstituted versions of biological membranes (e.g., giant unilamellar vesicles formed by lipids from brush border membranes (Dietrich et al., 2001) or by lung surfactants (Bernardino de la Serna et al., 2004) or, more recently, in vesicles obtained by various methods from cells (mast cells, fibroblasts, Baumgart et al., 2007; Sengupta et al., 2008; Veatch et al., 2008; A431 cells, Lingwood et al., 2008) and in budded HIV virus membranes (Brugger et al., 2006) (that are natural example of cell-originated membrane vesicles).

The properties of the lipid raft model inferred on the basis of these evidences are basically in agreement with those of the purified detergent-resistant membrane fractions (Sonnino and Prinetti, 2008) and describe membrane domains based on lipid-driven phase separation as relatively large, stable equilibrium structures, that can be affected by artificial or physiological perturbations. However, this scenario clashed with the view of lateral heterogeneity of biological membranes that emerged more recently by studies conducted using high-resolution (in time and space) techniques in unperturbed, living cells (that obviously represent the ultimate test bed for the lipid raft hypothesis) (reviewed in Jacobson et al., 2007; Lingwood and Simons, 2010).

In addition to fluorescence microscopy, that is particularly suitable for the study of living cells and is characterized by a high sensitivity, but hampered by a relatively poor spatial resolution, several high-resolution techniques have been used to study cell membrane heterogeneity in unperturbed cells (Table 1) (Jacobson et al., 2007; Owen et al., 2009). Some of these techniques are particularly appealing also for their capability to unveil the dynamics of membrane domains, that is completely lacking in equilibrium-based investigation methods, such as those used to study phase separation in model systems or those based on the analysis of membrane fractions separated on the basis of their detergent solubility.

When applied to the study of cell membrane heterogeneity, these techniques were supportive to the lipid raft hypothesis at a limited extent: they basically confirmed that there is a non-random distribution of cell surface molecules, leading to a highly hierarchical membrane organization. In addition, they confirmed the importance of cholesterol and sphingolipids in membrane domain formation (Eggeling et al., 2009). However, all these techniques measure experimental parameters that can be related to the association of a membrane molecule with a putative lipid raft, but basically no experimental technique is currently available to proof that membrane domains observed in intact cells correspond to a liquid-ordered phase. Indeed, the actual view depicted by the advent of these non-invasive approaches encompasses the existence of domains deeply differing in their size and spatial and temporal dynamics, but anyway strongly privileges the notion that membrane domains are small, highly dynamic structures, that are actively maintained and that can be generated, dissipated or deeply reorganized in response to diverse biochemical stimuli. The reported 
Table 1 | Some techniques used to the study of membrane heterogeneity in intact cells.

\begin{tabular}{|c|c|c|}
\hline Technique & Experimental observable & Selected references \\
\hline $\begin{array}{l}\text { Fluorescence recovery after photobleaching } \\
\text { (FRAP) }\end{array}$ & Translational mobility of a fluorophore & $\begin{array}{l}\text { Varma and Mayor (1998), Rao and } \\
\text { Mayor (2005) }\end{array}$ \\
\hline \multicolumn{3}{|l|}{ Fluorescence correlation spectroscopy (FCS) } \\
\hline Fluorescence resonance energy transfer (FRET) & $\begin{array}{l}\text { Energy transfer between an excited donor fluorophore } \\
\text { and an acceptor molecule, allowing to determine the } \\
\text { donor-acceptor proximity }\end{array}$ & Pralle et al. (2000) \\
\hline $\begin{array}{l}\text { Single fluorophore tracking microscopy (SFTM) } \\
\text { Single-particle fluorescence tracking (SPFT) } \\
\text { Single-particle tracking (SPT) }\end{array}$ & $\begin{array}{l}\text { Translational trajectories of membrane molecules } \\
\text { measuring the motility of a florescent label or of a } \\
\text { colloidal gold particle (in the latter case, by Raleigh light } \\
\text { scattering) specifically bound to the target molecule }\end{array}$ & $\begin{array}{l}\text { Saxton and Jacobson (1997), Jacobson } \\
\text { et al. (1995), Sheets et al. (1997), Chen } \\
\text { et al. (2006), Jacobson et al. (2007), } \\
\text { Jacobson and Dietrich (1999) }\end{array}$ \\
\hline $\begin{array}{l}\text { Stimulated emission depletion far-field } \\
\text { Fluorescence nanoscopy (STED) }\end{array}$ & $\begin{array}{l}\text { Time traces of single molecule diffusion of a } \\
\text { fluorescence-labeled probe }\end{array}$ & Eggeling et al. (2009) \\
\hline
\end{tabular}

size of these ordered structures in intact cells greatly varies between the nanometer (Wilson et al., 2000; Brugger et al., 2004; Lommerse et al., 2004a; Sharma et al., 2004; Douglass and Vale, 2005) and the micrometer-scale (Schutz et al., 2000; Hao et al., 2001; Gaus et al., 2003; Gomez-Mouton et al., 2004), and their lifespan ranges from microseconds (Dietrich et al., 2002; Dahan et al., 2003; Kusumi et al., 2005a) to milliseconds and seconds (Schutz et al., 2000; Gaus et al., 2003; Lommerse et al., 2004b; Douglass and Vale, 2005). These amazing differences in the reported features of membrane domains probably do at least in part reflect the great differences in spatial and temporal resolution that are characteristic of the different techniques used. On the other hand, it is unquestionable that they indicate the coexistence of multiple kinds of non-equilibrium membrane domains, whose features seem to be hardly explainable on the sole basis of the lipid raft hypothesis, i.e., on the basis of fluid-fluid phase separation.

Should we hence forget about liquid-ordered phase in real biological membranes? The answer is clearly no. The notion of liquid-ordered phase, and the derived concept of lipid raft, still explain several of the properties of membrane domains observed in intact cells, including the heterogeneity at the nanometer scale of membrane domains and their highly dynamic nature. For the latter point, it should be recalled that coalescence of lipid rafts, i.e., the fusion of nanoscale domains into larger signaling platforms, can occur at physiological temperature, possibly under physiologically relevant stimuli (Ayuyan and Cohen, 2008; Hofman et al., 2008; Lingwood et al., 2008), an indication that phase separation can occur under biologically relevant conditions in intact cells. Moreover, even if no analytical approach can identify a liquid-ordered phase in a living cells, convincing evidences on the ordered nature of lipid-driven membrane domains have been recently obtained. Two findings are particularly convincing in this direction: (1) stimulated emission depletion microscopy, that allows recording of membrane-associated molecules dynamics much below the diffraction limit imposed by visible light, showed the presence of cholesterol- and sphingolipid-dependent nanodomains that exclude glycerophospholipids and transiently trap GPI-anchored proteins (Eggeling et al., 2009); (2) Lingwood et al. (Lingwood et al., 2008) showed that, in plasma membrane spheres obtained by a cell-swelling procedure from A431 cells, cross-linking of GM1 ganglioside using pentavalent cholera toxin at $37^{\circ} \mathrm{C}$ resulted in the cholesterol-dependent coalescence of GM1-rich domains with the formation of micrometer-scale domains. The resulting GM1- and cholesterol-enriched phase is characterized by a lower translational diffusion, and fluorescent microscopy and spectroscopy performed using order-sensitive probes (Kaiser et al., 2009) revealed that the degree of lateral order in the GM1 domain is sensibly higher than in the surrounding membrane, however it is considerably lower than in the ordered phase of giant unilamellar vesicles, representative of a lipid ordered phase. As a consequence, this GM1 domains recruits lipid-anchored proteins usually regarded as lipid raft markers (but not transferrin receptor, usually excluded from lipid rafts), but also transmembrane proteins normally not associated with liquid-ordered phases in model systems or with detergentresistant membrane fraction.

From these studied clearly emerged that ordered phases in living cells resemble but are not completely equivalent to liquid-ordered phases, and that phase separation under physiological conditions only partially explains the behavior of ordered membrane domains, implying an important role for additional forces.

\section{LIPID-DEPENDENT MEMBRANE ORDER OTHER THAT FLUID-FLUID PHASE SEPARATION}

In cell membranes, in addition to liquid-liquid immiscibility, heterogeneity can be driven by a multitude of specific lateral interactions. It is a well established notion that proteins possess the potential to organize membrane domains, and indeed proteinprotein interactions have been regarded for several years as the main factor responsible for the stabilization of membrane macroand microdomains. Protein- and lipid-driven lateral organizations have been regarded as somehow mutually exclusive, while it is clear that they are cooperative in the creation of membrane structural and functional heterogeneity. Obvious limitations to reciprocal diffusion are present for proteins belonging to a multimolecular complex, formed by direct protein-protein interactions, such as 
the respiratory chain complex in prokaryotes and the complexes organized by membrane receptors. In addition, protein-driven membrane domains can be based on the presence of protein scaffolds (recently reviewed in Lindner and Naim, 2009), that can be organized by extracellular (e.g., galectins), intracellular (clathrin in clathrin-coated pits, the actin network at the cytosolic face of plasma membrane, that can be anchored to transmembrane proteins creating a "membrane-skeleton fence" limiting the lateral diffusion of molecules trapped in the fence (Kusumi and Suzuki, 2005b)), or membrane proteins [tetraspanins (Hemler, 2005), caveolins and cavins (Prinetti et al., 2008; Sonnino and Prinetti, 2009), flotillins (Rajendran et al., 2007)].

These domains are mainly driven in their formation and stabilized by protein-protein interactions, but specific binding between protein and lipids can be as well involved in the stabilization of the domains (Prinetti et al., 2009). Specific lipids are component of the quaternary structure of membrane-associated proteins or protein complexes, e.g., beta 2 adrenergic receptor (Cherezov et al., 2007; Hanson et al., 2008), cytochrome bc1 (Wenz et al., 2009), and specific binding of gangliosides to membrane receptors has been known from a long time, even if only recently some molecular details have been unveiled (reviewed in Prinetti et al., 2009). Some proteins that are associated with lipid domains are surrounded by a "shell" of typical raft lipids (sphingolipids, cholesterol) (Fantini and Barrantes, 2009): a lipid shell might confer to a membrane protein higher affinity for lipid rafts, determining its partitioning to a phase separated membrane domain in cooperation with or even in the absence of a specific raft targeting motif. Cholesterol binding domains and sphingolipid binding domains (that can bind the polar headgroups of sphingolipids) have been identified and characterized in several proteins (Epand, 2006; Fantini and Barrantes, 2009). The binding of lipids to receptors induces conformational changes affecting both ligand binding and signaling pathways downstream to receptor activation. On the other hand, it can deeply influence the lateral organization of membrane components in the domain of a membrane-organizing protein. For example caveolin-1, a typical scaffold-forming protein, tightly and specifically binds cholesterol (Murata et al., 1995; Li et al., 1996; Thiele et al., 2000), and cholesterol, in turn, is essential for caveolae formation and maintenance (Fielding and Fielding, 2000; Ikonen et al., 2004). Remarkably, palmitoylation (usually regarded as a lipid raft targeting motif) of caveolin does not affect its association with lipid rafts, however it is relevant for caveolin interaction with cholesterol (Uittenbogaard et al., 2002). The interaction of tetraspanins with cholesterol or sphingolipids does not affect direct interactions of tetraspanins with other membrane proteins

\section{REFERENCES}

Acquotti, D., Cantu, L., Ragg, E., and Sonnino, S. (1994). Geometrical and conformational properties of ganglioside GalNAcGD1a, IV4GalNAcIV3Neu5AcII3 Neu5AcGgOse4Cer. Eur. J. Biochem. 225, 271-288.

Acquotti, D., Fronza, G., Ragg, E., and Sonnino, S. (1991). Three

(integrins), but affects tetraspanins homo-oligomerization and signal transduction through tetraspanin-dependent complexes (glycosynapse) (Charrin et al., 2003; Hakomori, 2009).

Even in the case of membrane domains usually regarded as exclusively protein-driven and lipid raft-independent, a role of lipid-protein interactions has been described. In the case of clathrindependent endocytosis, phosphatidylinositol(4,5)-bisphosphate is used as the membrane anchor for several proteins involved in the formation of clathrin-coated pits (Haucke, 2005; Höning et al., 2005; Kusumi and Suzuki, 2005b). Transient confinement zones fenced by a diffusion barrier created by the anchoring of actin filaments to transmembrane proteins has been sometimes regarded as an alternative model to lipid rafts to explain the organization of membrane domains (Kusumi and Suzuki, 2005b). However, recent data indicate a close interplay between actin mediated- and lipid raft-mediated events (e.g., endocytosis of GPI-anchored proteins (Chadda et al., 2007; Goswami et al., 2008), and suggest that lipid-based domains can be stabilized by the cortical actin network (Chichili and Rodgers, 2009; Rollason et al., 2009).

\section{CONCLUSION}

One year before formalizing the fluid mosaic model, Singer and Nicolson (1971) wrote that, to fully understand the function of mammalian cell membranes, it would be necessary to know "the structure of cell membranes, we mean the detailed arrangement and conformation of the individual proteins, lipids, oligosaccharides and other components of membranes" and concluded that "we are a very long way from such knowledge at the present time." There is no doubt that tremendous progresses have been made in this direction, and our current view on membrane organization has been in the last decade deeply influenced by the lipid raft hypothesis. The lipid raft hypothesis had the great merit to re-focus the researcher's attention on the importance of lipids, and in particular on the importance of collective properties of a cellular lipid environment, in determining membrane organization, and to root the notion that membrane order is highly dynamic and changing. However it is gradually emerging the importance of the interplay of different lipid-sensitive mechanisms for membrane lateral organization, and an interpretation model solely based on fluid-fluid phase separation is probably no longer adequate in describing the whole complexity of lipid-dependent membrane heterogeneity.

\section{ACKNOWLEDGMENTS}

This work was supported by grants from CARIPLO, AIRC (to Sandro Sonnino) and the Mizutani Foundation for Glycoscience (to Alessandro Prinetti). The Authors thank Dr. Kazuhisa Iwabuchi for stimulating discussion.

oligosaccaride chain of GM1 ganglioside revealed by a distance-mapping procedure: a rotating and laboratory frame nuclear overhauser enhancement investigation of native glycolipid in dimethyl sulfoxide and in waterdodecylphosphocholine solutions. $J$. Am. Chem. Soc. 112, 7772-7778.

Almeida, P. F., Vaz, W. L., and Thompson, T. E. (1992). Lateral diffusion and percolation in two-phase, two-component lipid bilayers. Topology of the solid-phase domains in-plane and across the lipid bilayer. Biochemistry $31,7198-7210$.

Ayuyan, A. G., and Cohen, F. S. (2008) Raft composition at physiological temperature and $\mathrm{pH}$ in the absence of detergents. Biophys. J. 94, 2654-2666. 
Baumgart, T., Hammond, A. T., Sengupta, P., Hess, S. T., Holowka, D. A., Baird, B. A., and Webb, W. W. (2007). Largescale fluid/fluid phase separation of proteins and lipids in giant plasma membrane vesicles. Proc. Natl. Acad. Sci. U.S.A. 104, 3165-3170.

Baumgart, T., Hess, S. T., and Webb, W. W. (2003). Imaging coexisting fluid domains in biomembrane models coupling curvature and line tension. Nature 425, 821-824.

Bernardino de la Serna, J., Perez-Gil, J., Simonsen, A. C., and Bagatolli, L. A. (2004). Cholesterol rules: direct observation of the coexistence of two fluid phases in native pulmonary surfactant membranes at physiological temperatures. J. Biol. Chem. 279, 40715-40722.

Bouwstra, J.A., and Ponec, M. (2006). The skin barrier in healthy and diseased state. Biochim. Biophys. Acta 1758, 2080-2095.

Brocca, P., Acquotti, D., and Sonnino, S. (1996). Nuclear overhauser effect investigation on GM1 ganglioside containing $\mathrm{N}$-glycolyl-neuraminic acid (II3Neu5GcGgOse4Cer). Glycoconj. J. 13, 57-62.

Brocca, P., Berthault, P., and Sonnino, S. (1998). Conformation of the oligosaccharide chain of G(M1) ganglioside in a carbohydrate-enriched surface. Biophys. J. 74, 309-318.

Brocca, P., Cantu, L., and Sonnino, S. (1995). Aggregation properties of semisynthetic GD1a ganglioside (IV3Neu5AcII3Neu5AcGgOse4Cer) containing an acetyl group as acyl moiety. Chem. Phys. Lipids 77, 41-49.

Brown, D. A., and London, E. (2000). Structure and function of sphingolipid- and cholesterol-rich membrane rafts. J. Biol. Chem. 275, 17221-17224.

Brugger, B., Glass, B., Haberkant, P., Leibrecht, I., Wieland, F. T., and Krausslich, H. G. (2006). The HIV lipidome: a raft with an unusual composition. Proc. Natl. Acad. Sci. U.S.A. 103, 2641-2646.

Brugger, B., Graham, C., Leibrecht, I., Mombelli, E., Jen, A., Wieland, F., and Morris, R. (2004). The membrane domains occupied by glycosylphosphatidylinositol-anchored prion protein and Thy-1 differ in lipid composition. J. Biol. Chem. 279, 7530-7536.

Cantù, L., Corti, M., Casellato, R., Acquotti, D., and Sonnino, S. (1991). Aggregation properties of GD1b, II3Neu5Ac2GgOse4Cer, and of GD1blactone, II3[alpha-Neu5Ac-(2----8, 1----9)-alpha-Neu5Ac] GgOse4Cer, in aqueous solution. Chem. Phys. Lipids $60,111-118$.
Cantu, L., Corti, M., Sonnino, S., and Tettamanti, G. (1990). Evidence for spontaneous segregation phenomena in mixed micelles of gangliosides. Chem. Phys. Lipids 55, 223-229.

Chadda, R., Howes, M. T., Plowman, S. J., Hancock, J. F., Parton, R. G., and Mayor, S. (2007). Cholesterol-sensitive Cdc42 activation regulates actin polymerization for endocytosis via the GEEC pathway. Traffic 8, 702-717.

Charrin, S., Manie, S., Thiele, C., Billard, M., Gerlier, D., Boucheix, C., and Rubinstein, E. (2003). A physical and functional link between cholesterol and tetraspanins. Eur. J. Immunol. 33, 2479-2489.

Chen, Y., Lagerholm, B. C., Yang, B., and Jacobson, K. (2006). Methods to measure the lateral diffusion of membrane lipids and proteins. Methods 39 , 147-153.

Cherezov, V., Rosenbaum, D. M., Hanson, M. A., Rasmussen, S. G., Thian, F. S., Kobilka, T. S., Choi, H. J., Kuhn, P., Weis, W. I., Kobilka, B. K., and Stevens, R. C. (2007). High-resolution crystal structure of an engineered human beta2-adrenergic $\mathrm{G}$ protein-coupled receptor. Science 318, 1258-1265.

Chichili, G. R., and Rodgers, W. (2009). Cytoskeleton-membrane interactions in membrane raft structure. Cell. Mol. Life Sci. 66, 2319-2328.

Dahan, M., Levi, S., Luccardini, C. Rostaing, P., Riveau, B., and Triller, A. (2003). Diffusion dynamics of glycine receptors revealed by singlequantum dot tracking. Science 302, 442-445.

Del Favero, E., Brocca, P., Motta, S., Rondell, I.V., Sonnino, S., and Cantù, L. (2010). Nanoscale structural response of ganglioside-containing aggregates to the interaction with sialidase. $J$. Neurochem. doi: 10.1111/j.14714159.2010.07031.x.

Dietrich, C., Bagatolli, L.A., Volovyk,Z.N., Thompson, N. L., Levi, M., Jacobson, K., and Gratton, E. (2001). Lipid rafts reconstituted in model membranes. Biophys. J. 80, 1417-1428.

Dietrich, C., Yang, B., Fujiwara, T., Kusumi, A., and Jacobson, K. (2002). Relationship of lipid rafts to transient confinement zones detected by single particle tracking. Biophys. J. 82, 274-284.

Douglass, A. D., and Vale, R. D. (2005). Single-molecule microscopy reveals plasma membrane microdomains created by protein-protein networks that exclude or trap signaling molecules in T cells. Cell 121, 937-950.

Eggeling, C., Ringemann, C., Medda, R., Schwarzmann, G., Sandhoff, K., Polyakova, S., Belov, V. N., Hein, B., von Middendorff, C., Schönle, A., and Hell, S. W. (2009). Direct observation of the nanoscale dynamics of membrane lipids in a living cell. Nature 457 , 1159-1162.

Elson, E. L., Fried, E., Dolbow, J. E., and Genin, G. M. (2010). Phase separation in biological membranes: integration of theory and experiment. Аnпu. Rev Biophys. 39, 207-226.

Engelman, D. M. (1970). X-ray diffraction studies of phase transitions in the membrane of Mycoplasma laidlawii.J. Mol. Biol. 47, 115-117.

Epand, R. M. (2006). Cholesterol and the interaction of proteins with membrane domains. Prog. Lipid Res. 45 279-294.

Fantini, J., and Barrantes, F. J. (2009). Sphingolipid/cholesterol regulation of neurotransmitter receptor conformation and function. Biochim. Biophys. Acta 1788, 2345-2361.

Ferraretto, A., Pitto, M., Palestini, P. and Masserini, M. (1997). Lipid domains in the membrane: thermotropic properties of sphingomyelin vesicles containing GM1 ganglioside and cholesterol. Biochemistry 36 9232-9236.

Fielding, C. J., and Fielding, P. E. (2000). Cholesterol and caveolae: structural and functional relationships. Biochim. Biophys. Acta 1529, 210-222.

Gaus, K., Gratton, E., Kable, E. P., Jones, A. S., Gelissen, I., Kritharides, L., and Jessup, W. (2003). Visualizing lipid structure and raft domains in living cells with two-photon microscopy Proc. Natl. Acad. Sci. U.S.A. 100 15554-15559.

Goins, B., Masserini, M., Barisas, B. G., and Freire, E. (1986). Lateral diffusion of ganglioside GM1 in phospholipid bilayer membranes. Biophys. J. 49, 849-856.

Gomez-Mouton, C., Lacalle, R. A., Mira, E., Jimenez-Baranda, S., Barber, D. F., Carrera, A. C., Martinez, A. C., and Manes, S. (2004). Dynamic redistribution of raft domains as an organizing platform for signaling during cell chemotaxis. J. Cell Biol. 164 759-768.

Goni, F. M., Alonso, A., Bagatolli, L. A., Brown, R.E., Marsh, D., Prieto, M., and Thewalt, J. L. (2008). Phase diagrams of lipid mixtures relevant to the study of membrane rafts. Biochim. Biophys. Acta 1781, 665-684.

Gorter, E., and Grendel, F. (1925). On bimolecular layers of lipoids on the chromocytes of the blood. J. Exp. Med. 41, 439-443.

Goswami, D., Gowrishankar, K., Bilgrami, S., Ghosh, S., Raghupathy, R., Chadda, R., Vishwakarma, R., Rao, M., and Mayor, S. (2008). Nanoclusters of GPI-anchored proteins are formed by cortical actin-driven activity. Cell 135, 1085-1097.
Hakomori, S. (2004). Special issue on carbohydrate recognition through carbohydrate-carbohydrate interaction. Glycoconj. J. 21, 87.

Hakomori, S. I. (2009). Glycosynaptic microdomains controlling tumor cell phenotype through alteration of cell growth, adhesion and motility. FEBS Lett. 584, 1901-1906.

Hanson, M. A., Cherezov, V., Griffith, M. T., Roth, C. B., Jaakola, V. P., Chien, E. Y., Velasquez, J., Kuhn, P., and Stevens, R. C. (2008). A specific cholesterol binding site is established by the $2.8 \mathrm{~A}$ structure of the human beta2-adrenergic receptor. Structure 16, 897-905.

Hao, M., Mukherjee, S., and Maxfield, F. R. (2001). Cholesterol depletion induces large scale domain segregation in living cell membranes. Proc. Natl. Acad. Sci. U.S.A. 98, 13072-13077.

Haucke, V. (2005). Phosphoinositide regulation of clathrin-mediated endocytosis. Biochem. Soc. Trans. 33, 1285-1289.

Heatley, F., and Scott, J.E. (1988). A water molecule participates in the secondary structure of hyaluronan. Biochem. J. 254, 489-493.

Hemler, M. E. (2005). Tetraspanin functions and associated microdomains. Nat. Rev. Mol. Cell Biol. 6, 801-811.

Hofman, E. G., Ruonala, M. O., Bader, A. N., van den Heuvel, D., Voortman, J., Roovers, R. C., Verkleii, A. J., Gerritsen, H.C., and van Bergen En Henegouwen, P. M. (2008). EGF induces coalescence of different lipid rafts. J. Cell. Sci. 121, 2519-2528.

Holopainen, J. M., Metso, A. J., Mattila, J. P., Jutila, A., and Kinnunen, P. K. (2004). Evidence for the lack of a specific interaction between cholesterol and sphingomyelin. Biophys. J. $86,1510-1520$.

Höning, S., Ricotta, D., Krauss, M., Späte, K., Spolaore, B., Motley, A., Robinson, M.,Robinson,C.,Haucke, V., and Owen, D. J. (2005). Phosphatidylinositol(4,5)-bisphosphate regulates sorting signal recognition by the clathrinassociated adaptor complex AP2. Mol. Cell 18, 519-531.

Igbavboa, U., Avdulov, N. A., Chochina, S. V., and Wood, W. G. (1997) Transbilayer distribution of cholesterol is modified in brain synaptic plasma membranes of knockout mice deficient in the low-density lipoprotein receptor, apolipoprotein E, or both proteins. J. Neurochem. 69, 1661-1667.

Ikonen, E., Heino, S., and Lusa, S. (2004). Caveolins and membrane cholesterol. Biochem. Soc. Trans. 32, 121-123.

Ipsen, J. H., Karlstrom, G., Mouritsen, O. G., Wennerstrom, H., and 
Zuckermann, M. J. (1987). Phase equilibria in the phosphatidylcholinecholesterol system. Biochim. Biophys. Acta 905, 162-172.

Iwabuchi, K., Prinetti, A., Sonnino, S., Mauri, L., Kobayashi, T., Ishii, K., Kaga, N., Murayama, K., Kurihara, H., Nakayama,H., Yoshizaki, F., Takamori, K., Ogawa, H., and Nagaoka, I. (2008). Involvement of very long fatty acidcontaining lactosylceramide in lactosylceramide-mediated superoxide generation and migration in neutrophils. Glycoconj. J. 25, 357-374.

Jacobson, K., and Dietrich, C. (1999). Looking at lipid rafts? Trends Cell Biol. 9, 87-91.

Jacobson, K., Mouritsen, O. G., and Anderson, R. G. (2007). Lipid rafts: at a crossroad between cell biology and physics. Nat. Cell Biol. 9, 7-14.

Jacobson, K., Sheets, E. D., and Simson, R. (1995). Revisiting the fluid mosaic model of membranes. Science 268, 1441-1442.

Kahya, N., Scherfeld, D., and Schwille, P. (2005). Differential lipid packing abilities and dynamics in giant unilamellar vesicles composed of short-chain saturated glycerol-phospholipids, sphingomyelin and cholesterol. Chem. Phys. Lipids 135, 169-180.

Kaiser, H. J., Lingwood, D., Levental, I., Sampaio, J. L., Kalvodova, L., Rajendran, L., and Simons, K. (2009). Order of lipid phases in model and plasma membranes. Proc. Natl. Acad. Sci. U.S.A. 106, 16645-16650.

Karnovsky, M. J., Kleinfeld,A. M., Hoover, R. L., and Klausner, R. D. (1982). The concept of lipid domains in membranes. J. Cell Biol. 94, 1-6.

Klose, C., Ejsing, C. S., Garcia-Saez, A. J., Kaiser, H. J., Sampaio, J. L., Surma, M. A., Shevchenko, A., Schwille, P., and Simons, K. (2010). Yeast lipids can phase-separate into micrometer-scale membrane domains. J. Biol. Chem. 285, 30224-30232.

Kusumi, A., Nakada, C., Ritchie, K., Murase, K., Suzuki, K., Murakoshi, H., Kasai, R. S., Kondo, J., and Fujiwara, T. (2005a). Paradigm shift of the plasma membrane concept from the two-dimensional continuum fluid to the partitioned fluid: high-speed single-molecule tracking of membrane molecules. Annu. Rev. Biophys. Biomol. Struct. 34, 351-378.

Kusumi, A., and Suzuki, K. (2005b). Toward understanding the dynamics of membrane-raft-based molecular interactions. Biochim. Biophys. Acta 1746, 234-251

Lee, A. G., Birdsall, N. J., Metcalfe, J. C., Toon, P. A., and Warren, G. B. (1974). Clusters in lipid bilayers and the interpretation of thermal effects in biological membranes. Biochemistry 13, 3699-3705.

Levery, S. B. (1991). 1H-NMR study of GM2 ganglioside: evidence that an interresidue amide-carboxyl hydrogen bond contributes to stabilization of a preferred conformation. Glycoconj. J. 8, 484-492.

Li, S., Song, K. S., and Lisanti, M. P. (1996). Expression and characterization of recombinant caveolin. J. Biol. Chem. 271, 568-573.

Li, X. M., Momsen, M. M., Brockman, H. L., and Brown, R. E. (2003). Sterol structure and sphingomyelin acyl chain length modulate lateral packing elasticity and detergent solubility in model membranes. Biophys. J. 85, 3788-3801.

Lindner, R., and Naim, H. Y. (2009). Domains in biological membranes. Exp. Cell Res. 315, 2871-2878.

Lingwood, D., Ries, J., Schwille, P., and Simons, K. (2008). Plasma membranes are poised for activation of raft phase coalescence at physiological temperature. Proc. Natl. Acad. Sci. U.S.A. 105, 10005-10010.

Lingwood, D., and Simons, K. (2010). Lipid rafts as a membrane-organizing principle. Science 327, 46-50.

Lommerse, P. H., Blab, G. A., Cognet, L., Harms, G. S., Snaar-Jagalska, B. E., Spaink, H. P., and Schmidt, T. (2004a). Single-molecule imaging of the H-ras membrane-anchor reveals domains in the cytoplasmic leaflet of the cell membrane. Biophys. J. 86, 609-616.

Lommerse, P. H., Spaink, H. P., and Schmidt, T. (2004b). In vivo plasma membrane organization: results of biophysical approaches. Biochim. Biophys. Acta 1664, 119-131.

Masserini, M., and Freire, E. (1986) Thermotropic characterization of phosphatidylcholine vesicles containing ganglioside GM1 with homogeneous ceramide chain length. Biochemistry 25, 1043-1049.

Masserini, M., Palestini, P., and Freire E. (1989). Influence of glycolipid oligosaccharide and long-chain base composition on the thermotropic properties of dipalmitoylphosphatidylcholine large unilamellar vesicles containing gangliosides. Biochemistry 28, 5029-5034

Masserini, M., Palestini, P., Venerando, B., Fiorilli, A., Acquotti, D., and Tettamanti, G. (1988). Interactions of proteins with ganglioside-enriched microdomains on the membrane: the lateral phase separation of molecular species of GDla ganglioside, having homogeneous long-chain base composition, is recognized by Vibrio cholerae sialidase. Biochemistry 27, 7973-7978.
Mattjus, P., and Slotte, J. P. (1996). Does cholesterol discriminate between sphingomyelin and phosphatidylcholine in mixed monolayers containing both phospholipids? Chem. Phys. Lipids 81, 69-80.

Mayor, S., and Rao, M. (2004). Rafts: scaledependent, active lipid organization at the cell surface. Traffic 5, 231-240.

Mombelli, E., Morris, R., Taylor, W. and Fraternali, F. (2003). Hydrogenbonding propensities of sphingomyelin in solution and in a bilayer assembly: a molecular dynamics study. Biophys. J. 84, 1507-1517.

Mouritsen, O. G. (2010). The liquidordered state comes of age. Biochim. Biophys. Acta 1798, 1286-1288.

Murata, M., Peranen, J., Schreiner, R. Wieland, F., Kurzchalia, T. V., and Simons, K. (1995). VIP21/caveolin is a cholesterol-binding protein. Proc. Natl Acad. Sci. U.S.A. 92, 10339-10343.

Ohvo-Rekila, H., Ramstedt, B., Leppimaki, P., and Slotte, J. P. (2002). Cholesterol interactions with phospholipids in membranes. Prog. Lipid Res. 41 , 66-97.

Owen, D. M., Williamson, D., Rentero, C., and Gaus, K. (2009). Quantitative microscopy: protein dynamics and membrane organisation. Traffic 10 962-971.

Palestini, P., Allietta, M., Sonnino, S., Tettamanti, G., Thompson, T. E., and Tillack, T. W. (1995). Gel phase preference of ganglioside GM1 at low concentration in two-component, two-phase phosphatidylcholine bilayers depends upon the ceramide moiety. Biochim. Biophys. Acta 1235 221-230.

Palestini, P., Masserini, M., and Tettamanti, G. (1994). Exposure to galactose oxidase of GM1 ganglioside molecular species embedded into phospholipid vesicles. FEBS Lett. 350, 219-222.

Pandit, S. A., Jakobsson, E., and Scott, H. L (2004). Simulation of the early stages of nano-domain formation in mixed bilayers of sphingomyelin, cholesterol, and dioleylphosphatidylcholine. Biophys. J. 87, 3312-3322.

Pascher, I. (1976). Molecular arrangements in sphingolipids. Conformation and hydrogen bonding of ceramide and their implication on membrane stability and permeability. Biochim. Biophys. Acta 455, 433-451.

Pitto, M., Parenti, M., Guzzi, F., Magni, F. Palestini, P., Ravasi, D., and Masserini, M. (2002). Palmitic is the main fatty acid carried by lipids of detergentresistant membrane fraction from neural and non-neural cells. Neurochem. Res. 27, 729-734

Poppe, L., van Halbeek, H., Acquotti, D., and Sonnino, S. (1994). Carbohydrate dynamics at a micellar surface: GD1a headgroup transformations revealed by NMR spectroscopy. Biophys. J. 66, 1642-1652.

Pralle, A., Keller, P., Florin, E. L., Simons, K., and Horber, J. K. (2000). Sphingolipid-cholesterol rafts diffuse as small entities in the plasma membrane of mammalian cells. J. Cell Biol. 148, 997-1008.

Prinetti, A., Chigorno, V., Prioni, S., Loberto, N., Marano, N., Tettamanti, G., and Sonnino, S. (2001). Changes in the lipid turnover, composition, and organization, as sphingolipid-enriched membrane domains, in rat cerebellar granule cells developing in vitro. $J$. Biol. Chem. 276, 21136-21145.

Prinetti, A., Loberto, N., Chigorno, V., and Sonnino, S. (2009). Glycosphingolipid behaviour in complex membranes. Biochim. Biophys. Acta 1788 184-193.

Prinetti, A., Prioni, S., Loberto, N., Aureli, M., Chigorno, V., and Sonnino, S. (2008). Regulation of tumor phenotypes by caveolin-1 and sphingolipid-controlled membrane signaling complexes. Biochim. Biophys. Acta 1780, 585-596.

Quinn, P. J. (2010). A lipid matrix model of membrane raft structure. Prog. Lipid Res. 49, 390-406.

Quinn, P. J., and Wolf, C. (2009). The liquid-ordered phase in membranes. Biochim. Biophys. Acta 1788, 33-46.

Rajendran, L., Le Lay, S., and Illges, H. (2007). Raft association and lipid droplet targeting of flotillins are independent of caveolin. Biol. Chem. 388, 307-314.

Rao, M., and Mayor, S. (2005). Use of Forster's resonance energy transfer microscopy to study lipid rafts. Biochim. Biophys. Acta 1746, 221-233.

Rock, P., Allietta, M., Young, W. W. Jr., Thompson, T. E., and Tillack, T. W. (1991). Ganglioside GM1 and asialoGM1 at low concentration are preferentially incorporated into the gel phase in two-component, two-phase phosphatidylcholine bilayers. Biochemistry 30, 19-25.

Rollason, R., Korolchuk, V., Hamilton, C., Jepson, M., and Banting, G. (2009). A CD317/tetherin-RICH2 complex plays a critical role in the organization of the subapical actin cytoskeleton in polarized epithelial cells. J. Cell Biol. 184, 721-736.

Ruettinger, A., Kiselev, M. A., Hauss, T., Dante, S., Balagurov, A. M., and Neubert, R. H. (2008). Fatty acid interdigitation in stratum corneum model membranes: a neutron diffraction study. Eur. Biophys. J.37, 759-771. 
Sankaram, M. B., and Thompson, T. E. (1990). Interaction of cholesterol with various glycerophospholipids and sphingomyelin. Biochemistry 29, 10670-10675.

Saxton, M. J., and Jacobson, K. (1997). Single-particle tracking: applications to membrane dynamics. Annu. Rev. Biophys. Biomol. Struct. 26, 373-399.

Scarsdale, J. N., Prestegard, J. H., and Yu, R. K. (1990). NMR and computational studies of interactions between remote residues in gangliosides. Biochemistry 29, 9843-9855.

Schroeder, F., Nemecz, G., Wood, W. G., Joiner, C., Morrot, G., Ayraut-Jarrier, M., and Devaux, P. F. (1991). Transmembrane distribution of sterol in the human erythrocyte. Biochim. Biophys. Acta 1066, 183-192.

Schutz, G. J., Kada, G., Pastushenko, V. P., and Schindler, H. (2000). Properties of lipid microdomains in a muscle cell membrane visualized by single molecule microscopy. EMBO J. 19, 892-901.

Sengupta, P., Hammond, A., Holowka, D., and Baird, B. (2008). Structural determinants for partitioning of lipids and proteins between coexisting fluid phases in giant plasma membrane vesicles. Biochim. Biophys. Acta 1778, 20-32.

Sharma, P., Varma, R., Sarasij, R. C., Ira, Gousset, K., Krishnamoorthy, G., Rao, M., and Mayor, S. (2004). Nanoscale organization of multiple GPI-anchored proteins in living cell membranes. Cell 116, 577-589.

Sheets, E. D., Lee, G. M., Simson, R., and Jacobson, K. (1997). Transient confinement of a glycosylphosphatidylinositol-anchored protein in the plasma membrane. Biochemistry 36, 12449-12458.

Siebert, H. C., Reuter, G., Schauer, R., von der Lieth, C. W., and Dabrowski, J. (1992). Solution conformations of GM3 gangliosides containing different sialic acid residues as revealed by NOE-based distance mapping, molecular mechanics, and molecular dynamics calculations. Biochemistry 31, 6962-6971.
Simons, K., and van Meer, G. (1988). Lipid sorting in epithelial cells. Biochemistry 27, 6197-6202.

Singer, S. J., and Nicolson, G. L. (1971). The structure and chemistry of mammalian cell membranes. Am. J. Pathol. 65, 427-437.

Singer, S. J., and Nicolson, G. L. (1972). The fluid mosaic model of the structure of cell membranes. Science 175, 720-731.

Snyder, B., and Freire, E. (1980). Compositional domain structure in phosphatidylcholine--cholesterol and sphingomyelin--cholesterol bilayers. Proc. Natl. Acad. Sci. U.S.A. 77, 4055-4059.

Sonnino, S., Cantu, L., Acquotti, D., Corti, M., and Tettamanti, G. (1990a). Aggregation properties of GM3 ganglioside (II3Neu5AcLacCer) in aqueous solutions. Chem. Phys. Lipids 52, 231-241.

Sonnino, S., Cantu, L., Corti, M. Acquotti, D., Kirschner, G., and Tettamanti, G. (1990b). Aggregation properties of semisynthetic GM1 ganglioside (II3Neu5AcGgOse4Cer) containing an acetyl group as acyl moiety. Chem. Phys. Lipids 56, 49-57.

Sonnino, S., Cantu, L., Corti, M., Acquotti, D., and Venerando, B. (1994). Aggregative properties of gangliosides in solution. Chem. Phys. Lipids 71, 21-45.

Sonnino, S., Mauri, L., Chigorno, V., and Prinetti, A. (2007). Gangliosides as components of lipid membrane domains. Glycobiology 17, 1R-13R.

Sonnino, S., and Prinetti, A. (2008). Membrane lipid domains and membrane lipid domain preparations: are they the same thing? Trends Glycosci. Glycotechnol. 20, 315-340.

Sonnino, S., and Prinetti, A. (2009). Sphingolipids and membrane environments for caveolin. FEBS Lett. 583, 597-606.

Sonnino, S., Prinetti, A., Mauri, L., Chigorno, V., and Tettamanti, G. (2006). Dynamic and structural properties of sphingolipids as driving forces for the formation of membrane domains. Chem. Rev. 106, 2111-2125.
Steim, J. M., Tourtellotte, M. E., Reinert, J. C., McElhaney, R. N., and Rader, R. L. (1969). Calorimetric evidence for the liquid-crystalline state of lipids in a biomembrane. Proc. Natl. Acad. Sci. U.S.A. 63, 104-109.

Terzaghi, A., Tettamanti, G., and Masserini, M. (1993). Interaction of glycosphingolipids and glycoproteins: thermotropic properties of model membranes containing GM1 ganglioside and glycophorin. Biochemistry 32 9722-9725.

Thiele, C., Hannah, M. J., Fahrenholz, F. and Huttner, W. B. (2000). Cholesterol binds to synaptophysin and is required for biogenesis of synaptic vesicles. Nat. Cell Biol. 2, 42-49.

Tian, A., and Baumgart, T. (2009). Sorting of lipids and proteins in membrane curvature gradients. Biophys. J. 96, 2676-2688.

Tourtellotte, M. E., Branton, D., and Keith, A. (1970). Membrane structure: spin labeling and freeze etching of Mycoplasma laidawii. Proc. Natl. Acad. Sci. U.S.A. 66, 909-916.

Uittenbogaard, A., Everson, W. V., Matveev, S. V., and Smart, E. J. (2002) Cholesteryl ester is transported from caveolae to internal membranes as part of a caveolin-annexin II lipidprotein complex. J. Biol. Chem. 277, 4925-4931.

Varma, R., and Mayor, S. (1998). GPIanchored proteins are organized in submicron domains at the cell surface. Nature 394, 798-801.

Veatch, S. L., Cicuta, P., Sengupta, P. Honerkamp-Smith, A., Holowka, D., and Baird, B. (2008). Critical fluctuations in plasma membrane vesicles. ACS Chem. Biol. 3 , 287-293.

Veatch, S. L., and Keller, S. L. (2005). Miscibility phase diagrams of giant vesicles containing sphingomyelin. Phys. Rev. Lett. 94, 148101.

Wenz, T., Hielscher, R., Hellwig, P. Schagger, H., Richers, S., and Hunte, C. (2009). Role of phospholipids in respiratory cytochrome bc(1) complex catalysis and supercomplex formation. Biochim. Biophys. Acta 1787 609-616.
Westerlund, B., and Slotte, J. P. (2009). How the molecular features of glycosphingolipids affect domain formation in fluid membranes. Biochim. Biophys. Acta 1788, 194-201.

Wilson, B. S., Pfeiffer, J. R., and Oliver, J. M. (2000). Observing FcepsilonRI signaling from the inside of the mast cell membrane. J. Cell Biol. 149, 1131-1142.

Wunderlich, F., Kreutz, W., Mahler, P., Ronai, A., and Heppeler, G. (1978). Thermotropic fluid goes to ordered "discontinuous" phase separation in microsomal lipids of Tetrahymena. An $\mathrm{X}$-ray diffraction study. Biochemistry 17, 2005-2010.

Wunderlich, F., Ronai, A., Speth, V., Seelig, J., and Blume, A. (1975). Thermotropic lipid clustering in tetrahymena membranes. Biochemistry 14, 3730-3735.

Yoshizaki, F., Nakayama, H., Iwahara, C., Takamori,K.,Ogawa,H., and Iwabuchi, K. (2008). Role of glycosphingolipidenriched microdomains in innate immunity: microdomain-dependent phagocytic cell functions. Biochim. Biophys. Acta 1780, 383-392.

Conflict of Interest Statement: The authors declare that the research was conducted in the absence of any commercial or financial relationships that could be construed as a potential conflict of interest.

Received: 30 September 2010; paper pending published: 22 October 2010; accepted: 28 October 2010; published online: 19 November 2010.

Citation: Sonnino S and Prinetti A (2010) Lipids and membrane lateral organization. Front. Physio. 1:153. doi: 10.3389/ fphys.2010.00153

This article was submitted to Frontiers in Membrane Physiology and Biophysics, a specialty of Frontiers in Physiology. Copyright (C) 2010 Sonnino and Prinetti. This is an open-access article subject to an exclusive license agreement between the authors and the Frontiers Research Foundation, which permits unrestricted use, distribution, and reproduction in any medium, provided the original authors and source are credited. 